\title{
Calorimetric and Compressibility Study of Aqueous Solutions of Dextran with Special Reference to Hydration and Structural Change of Water
}

\author{
Fumio KaWAIZUmi, Noriyuki Nishio, Hiroyasu NomURA, \\ and Yutaka MiYAHARA \\ Department of Chemical Engineering, Faculty of Engineering, Nagoya University, \\ Furo-cho, Chikusa-ku, Nagoya 464, Japan.
}

(Received June 21, 1980)

\begin{abstract}
Interactions between water and dextran have been investigated by measuring specific heat capacity, adiabatic compressibility, and viscosity, with dextran samples of molecular weight above $10^{4}$. For these measurements, a laboratory-constructed isoperibol twin calorimeter and an ultrasonic interferometer were used. The compressibility data show the existence of the water hydrated to dextran molecules, and the amount of hydration water was determined. The partial molar heat capacity was calculated for the structural unit of the dextran molecule. In the plot of $\log$ [ $\eta]$ against $\log M_{w}$, a downward deviation from the linear relation was observed, and this was interpreted as due to branching of the dextran molecule. The amount of hydration water and the partial molar heat capacity decreased with the increasing molecular weight of dextran. The quantity $\Delta \bar{C}_{p_{2}}{ }^{\circ}=\bar{C}_{p_{2}}{ }^{\circ}-C_{p_{2}}$ (pure state) indicates that the structural change of water caused by the dissolution of one structural unit of the dextran molecule is smaller than in the case of glucose and that the degree of the structural change of water decreases sharply with the increasing molecular weight of polymers. These results are interpreted in terms of the combination of branching and increased entanglement of the polymer chain.

KEY WORDS Partial Molar Heat Capacity / Compressibility / Dextran /

Partial Specific Compression / Hydration / Aqueous Solution / Structure of Water /
\end{abstract}

Dextran is an anhydroglucose polymer mainly consisting of $\alpha$-1,6-glucosidic linkages, and dissolves readily in water. Its solution properties have called not only the attention of polymer scientists but also that of biochemists. To obtain information about the interactions of dissolved dextran molecules in aqueous solution, many techniques have been applied, such as measurements of adiabatic compressibility, ${ }^{1,2}$ viscosity, ${ }^{2-4}$ light scattering, $^{3}$ sedimentation velocity, ${ }^{3,4}$ and ultrasonic absorption coefficients. ${ }^{5}$ Earlier studies ${ }^{3,4}$ have been discussed by Gekko and Noguchi ${ }^{2}$ in connection with their study of oligodextran. Most workers have been interested in the conformation of the dissolved dextran molecules, but the discussion on the structural change of solvent water have often been ignored. In view of this situation, we have undertaken a determination of adiabatic compressibility, viscosity, and partial specific volume of dextran in addition to that of the partial molar heat capacity of the aqueous solutions of dextran. The partial molar heat capacity of a solute is that quantity which reflects the structural change in the solvent. Dextran samples of molecular weight above $10^{4}$ were used.

\section{EXPERIMENTAL}

\section{Dextran}

The specimens used were supplied by Pharmacia Fine Chemical (Sweden). Table $\mathrm{I}$ is a reproduction of the data sheet supplied. In response to our request, the comment was given from the agency, Pharmacia Japan Co. that these specimens were produced by the B-512 strain of Leuconostoc mensenteroides and were purified by the fractional precipitation using ethanol. This strain is the same 
Table I. Molecular weights of the dextrans used and the values of $[\eta]$ in water given in the supplied data sheet

\begin{tabular}{lllcll}
\hline & T 10 & T 40 & T 110 & T 500 & T 2000 \\
\hline$M_{w} / 10^{4}$ & 0.94 & 4.0 & 11 & 50 & 200 \\
$M_{n} / 10^{4}$ & 0.55 & 3.0 & 7.6 & $35^{\mathrm{a}}$ & $143^{\mathrm{a}}$ \\
{$[\eta]\left(20^{\circ} \mathrm{C}\right)$} & 0.096 & 0.21 & 0.32 & - & 0.70 \\
\hline
\end{tabular}

a Estimated by us from the ratio of $M_{w} / M_{n}$ for T 110 .

as that used by Senti et al. ${ }^{3}$ and Granath. ${ }^{4}$ Dextran is hygroscopic so that before use, the samples were dehydrated in a vacuum electric oven at $90-95^{\circ} \mathrm{C}$.

The water content of the original samples was in the range of about $5-10 \mathrm{wt} \%$.

\section{Apparatus and Procedures}

The adiabatic compressibility of aqueous solutions of dextran was determined by measuring the ultrasonic velocity and density. An improved ultrasonic interferometer working at $4 \mathrm{MHz}$ was used. The density was determined by an Ostwaldtype pycnometer of $20 \mathrm{~cm}^{3}$ capacity. The specific heat capacity of the solution was measured using an isoperibol twin calorimeter. The details of this calorimeter and the experimental procedures of the solution calorimetry are described elsewhere., Viscosity was measured using an Ubbelohde-type viscometer and the intrinsic viscosity $[\eta]$ was determined by the usual method. Determination of the specific heat capacity of the solution was carried out at about $30^{\circ} \mathrm{C}$, while other properties were measured at $25^{\circ} \mathrm{C}$.

\section{RESULTS AND DISCUSSION}

Results of viscosity measurements on aqueous solutions of dextran are shown in Figure 1, where $\log [\eta]$ is plotted agaisnt $\log M_{w}$. Our results agree satisfactorily with those of Senti et al. ${ }^{3}$ who obtained the relation $[\eta]=9.78 \times 10^{-4} M_{w}{ }^{0.50}$ for fractions of dextran with molecular weights below about $10^{5}$ (the dotted line in Figure 1). Senti et al. argued that a downward deviation from the linear relation is attributable to the branching of dextran molecules. It has been observed that more highly branched dextran molecules have smaller intrinsic viscosities. ${ }^{4}$ If the number-average molecular weight $M_{n}$ is used in place of $M_{w}$, Senti's relation reduces to

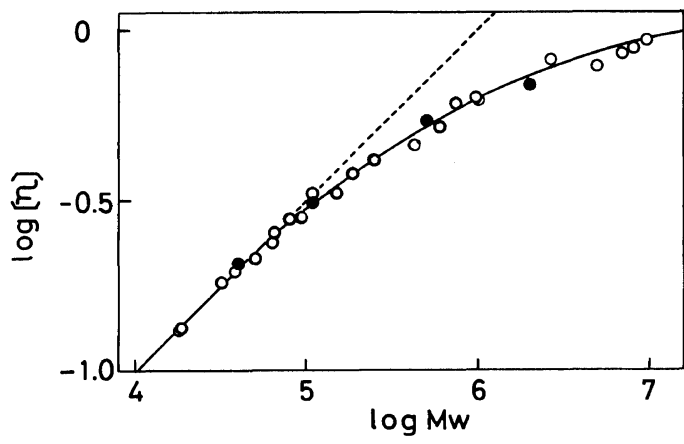

Figure 1. Double-logarithmic plot of intrinsic viscosity, [ $\eta$ ], against $M_{w}$ for dextran in water at $25^{\circ} \mathrm{C}$ : present work; $\bigcirc$, Senti et $a .^{3}$

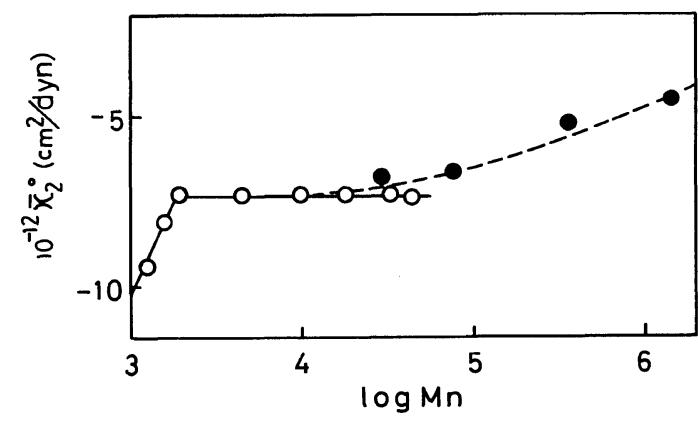

Figure 2. Molecular weight dependence of partial specific compressibility of dextran in water at $25^{\circ} \mathrm{C}$ : present work; $\bigcirc$, Gekko and Noguchi. ${ }^{2}$

$[\eta]=1.09 \times 10^{-3} M_{n}^{0.5}$, while Gekko and Noguchi, ${ }^{2}$ using dextran samples supplied by the Central Research Institute of Meito Sangyo Co. have obtained the relation $[\eta]=4.93 \times 10^{-4} M_{n}{ }^{0.60}$ for oligodextran of $M_{n}$ between 2000 and $4.5 \times 10^{4}$. The differences observed between these two measurements ${ }^{2,4}$ are significant. Therefore, it seems that the characteristics of two kinds of dextrans are different to some extent. In what follows, a comparison will be presented between the results of Gekko and Noguchi ${ }^{2}$ and ours but a part of the observed differences arises from the differences in the specimens used.

The limiting partial specific adiabatic compressibility of a solute, $\bar{\kappa}_{2}^{\circ}$, was obtained by extrapolating the data for the compressibility and density of the solution to infinite dilution. ${ }^{8} \bar{\kappa}_{2}{ }^{\circ}$ is defined by

$$
\bar{\kappa}_{2}^{\circ}=-\frac{\kappa_{1}}{\bar{v}_{2}{ }^{\circ}}\left(\frac{\partial \bar{v}_{2}}{\partial p}\right)^{0}
$$


where $\bar{v}_{2}^{\circ}$ is the partial specific volume of the solute, $p$ is the pressure and the superscript zero refers to infinite dilution. The variation of $\bar{\kappa}_{2}{ }^{\circ}$ with $M_{n}$ is illustrated in Figure 2, together with the results given by Gekko and Noguchi. ${ }^{2}$ In correspondence with the results from viscosity measurements, for $M_{n}$ above $5 \times 10^{4}$, the values of $\bar{\kappa}_{2}^{\circ}$ deviate from a constant value of $-7.3 \times 10^{-12} \mathrm{~cm}^{2} \mathrm{dyn}^{-1}$ obtained for $M_{n}$ between $2 \times 10^{3}$ and $5 \times 10^{4}$, and increase with $M_{n}$. Since a value of about $20 \times 10^{-12} \mathrm{~cm}^{2}$ $\mathrm{dyn}^{-1}$ is almost the lower limit for "normal" polymers such as polystyrene and poly(vinyl acetate) in very poor solvents, the negative values for dextran in water indicate a marked compacting of the polymer by the immobilization of the neighbouring water molecules (hydration). The amount of hydration water can be estimated by assuming that the solute is incompressible and that the compressibility of hydration water is identical with that of ice, $18 \times 10^{-12} \mathrm{~cm}^{2} \mathrm{dyn}^{-1} .^{9}$ The amount of hydration water, $\omega$, is proportional to $\bar{\kappa}_{2}^{\circ}$, $\left(\omega=-\bar{v}_{2}^{\circ} \bar{\kappa}_{2}^{\circ} /\left(\kappa_{1}-\kappa_{\text {ice }}\right)\right)$, so that the dependence of the amount of hydration on the molecular weight of dextran is the same as that of $\bar{\kappa}_{2}^{\circ}$. Hydration decreases with increasing molecular weight of dextran. The amount of hydration water thus determined is given in the fourth column of Table III.

Experimental results of solution calorimetry of dextran are given in Table II. In this table, the structural unit of dextran

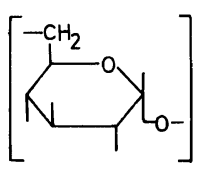

$=162.1$ was taken to calculate molecular weights, with the end effect and branching of the polymer chain being neglected. The partial molar heat capacity of the aqueous solution of dextran at infinite dilution, $\overline{C_{p_{2}}}$, was calculated by use of the expression, $m \phi_{C_{p}}=b m+c m^{2}$, where $\phi_{C_{p}}$ is the apparent molar heat capacity.

The quantity $m \phi_{C_{p}}$ was expressed in terms of the

Table II. Specific heat capacities of aqueous solutions of dextrans at $c a .303 \mathrm{~K}$

(a) Dextran $\mathrm{T} 10$

\begin{tabular}{cccc}
\hline$m$ & \multicolumn{1}{c}{$C_{p}$} & & $m \phi_{C p}$ \\
$\mathrm{~mol} \mathrm{~kg}^{-1}$ & & $\mathrm{~J} \mathrm{~g}^{-1} \mathrm{~K}^{-1}$ & $\mathrm{~J} \mathrm{~K}^{-1}$ \\
\hline 0.4472 & 4.009 & 121 \\
0.5743 & 3.968 & 159 \\
0.6834 & 3.938 & 196 \\
0.7701 & 3.914 & 224 \\
0.8939 & 3.879 & 262 \\
1.0189 & 3.838 & 294 \\
1.1423 & 3.805 & 332 \\
\hline
\end{tabular}

(b) Dextran $\mathrm{T} 40$

\begin{tabular}{cccc}
\hline$m$ & \multicolumn{1}{c}{$C_{p}$} & & $m \phi_{C p}$ \\
$\mathrm{~mol} \mathrm{~kg}^{-1}$ & & $\mathrm{~J} \mathrm{~g}^{-1} \mathrm{~K}^{-1}$ & \\
\hline 0.3367 & 4.050 & \\
0.4509 & 4.009 & 92.3 \\
0.5610 & 3.967 & 123 \\
0.6810 & 3.926 & 150 \\
0.7913 & 3.890 & 181 \\
0.9000 & 3.852 & 210 \\
1.0003 & 3.821 & 236 \\
\hline
\end{tabular}

(c) Dextran $\mathrm{T} 110$

\begin{tabular}{cccc}
\hline$m$ & \multicolumn{1}{c}{$C_{p}$} & & $m \phi_{C p}$ \\
$\mathrm{~mol} \mathrm{~kg}^{-1}$ & & $\mathrm{~J} \mathrm{~g}^{-1} \mathrm{~K}^{-1}$ & $\mathrm{~J} \mathrm{~K}^{-1}$ \\
\hline 0.1171 & 4.128 & 27.7 \\
0.2161 & 4.086 & 50.3 \\
0.3284 & 4.046 & 83.1 \\
0.4317 & 4.008 & 110 \\
0.5406 & 3.967 & 136 \\
0.6661 & 3.931 & 177 \\
0.7668 & 3.879 & 183 \\
\hline
\end{tabular}

(d) Dextran T 2000

\begin{tabular}{|c|c|c|}
\hline$m$ & $C_{p}$ & $m \phi_{C_{p}}$ \\
\hline $\mathrm{mol} \mathrm{kg}^{-1}$ & $\mathrm{~J} \mathrm{~g}^{-1} \mathrm{~K}^{-1}$ & $\mathrm{~J} \mathrm{~K}^{-1}$ \\
\hline 0.1124 & 4.132 & 29.0 \\
\hline 0.2348 & 4.079 & 55.6 \\
\hline 0.3430 & 4.027 & 72.0 \\
\hline 0.4788 & 3.970 & 99.4 \\
\hline 0.5756 & 3.947 & 137 \\
\hline 0.6880 & 3.906 & 163 \\
\hline 0.8383 & 3.786 & 122 \\
\hline
\end{tabular}


Table III. Physical parameters of dextrans in water

\begin{tabular}{|c|c|c|c|c|c|c|}
\hline Snecimen & [n] & $\bar{v}_{2}^{\circ}$ & $\omega$ & $\bar{\kappa}_{2}{ }^{\circ}$ & ${\overline{C_{p_{2}}}}^{\circ}$ & $\Delta{\overline{C_{p_{2}}}}^{\circ}$ \\
\hline & & $\mathrm{cm}^{3} \mathrm{~g}^{-1}$ & $\mathrm{~cm}^{3} \mathrm{~g}^{-1}$ & $10^{-12} \mathrm{~cm}^{2} \mathrm{dyn}^{-1}$ & $\mathrm{~J} \mathrm{~mol}^{-1} \mathrm{~K}^{-1}$ & $\mathrm{~J} \mathrm{~mol}{ }^{-1} \mathrm{~K}^{-1}$ \\
\hline T 10 & - & - & - & - & $272 \pm 6$ & 73 \\
\hline T 40 & 0.206 & 0.616 & 0.16 & -6.8 & $278 \pm 2$ & 79 \\
\hline T 110 & 0.311 & 0.612 & 0.15 & -6.6 & $259 \pm 13$ & 60 \\
\hline T 500 & 0.541 & 0.620 & 0.12 & -5.2 & - & - \\
\hline T 2000 & 0.691 & 0.614 & 0.10 & -4.5 & $202 \pm 16$ & 3 \\
\hline
\end{tabular}

quadratic form of the molal concentation $m$ to avoid the overemphasis of the data obtained in dilute region where the $C_{p}$ values are less accurate.

All the results obtained in the present work are summarized in Table III, in which $\Delta \bar{C}_{p_{2}}{ }^{\circ}$ is defined as $\Delta{\overline{C_{p_{2}}}}^{\circ}=C_{p_{2}}{ }^{\circ}-C_{p_{2}}$ (pure state). As mentioned above, the values of ${\overline{C_{p_{2}}}}^{\circ}$ shown in Table III were calculated for the structural unit of the dextran molecule. The hypothetical value of the heat capacity of the structural unit of the dextran molecule in the pure state was estimated by modifying the method proposed by Dobratz. ${ }^{10}$ The estimated value is $199 \mathrm{~J} \mathrm{~mol}^{-1} \mathrm{~K}^{-1}$. This value permits the calculation of $\Delta \overline{C_{p_{2}}}{ }^{\circ}$ given in the last column of Table III. The quantity $\Delta \overline{C_{p_{2}}}{ }^{\circ}$ is a measure of the change in the water structure originating from the dissolution of solutes.

The monomer of the dextran molecule is glucose, for which the following values have been reported (the same system of units as in Table III is adopted):

\begin{tabular}{|c|c|c|c|c|}
\hline $\bar{v}_{2}^{\circ}$ & & $\omega$ & $C_{p_{2}}{ }^{\circ}$ & $\Delta C_{p_{2}}^{\circ}$ \\
\hline 0.621 & $0.35,^{9}$ & $0.37^{11}$ & $336^{7}$ & $109^{7}$ \\
\hline
\end{tabular}

In Table III, the value of $\bar{v}_{2}^{\circ}$ remains constant within the limit of experimental error, and this value coincides with that for the glucose molecule shown above. The degree of polymerization has little effect on the partial specific volume of dextran in water. On the other hand, the amount of bound water calculated from the partial specific compressibility decreases with increasing molecular weight. It is reasonable to attribute the hydration of dextran is attributable to the hydrophilic $\mathrm{OH}$ group contained in the molecule. The decrease of the hydration water determined as the unit quantity of polymer as function of molecular weight of dextran may be due to the decrease in the number of the free $\mathrm{OH}$ groups contacting directly the surrounding water molecules.

The values of $\Delta \bar{C}_{p_{2}} \circ$ decrease as a result of the decrease in $\overline{C_{p_{2}}}$. values with molecular weight of dextran. A comparison of $\Delta \overline{C_{p_{2}}}$ of dextran with the corresponding value for glucose indicates that the change in the water structure caused by dissolution of one structural unit of the dextran molecule is smaller than in the case of glucose. In addition, the degree of this change decreases rapidly with increasing molecular weight of polymer, and for dextran T 2000, $\overline{C_{p_{2}}} \circ$ and $C_{p_{2}}$ (pure state) are nearly the same in magnitude.

The deviation from the linearity in the doublelogarithmic plot of $[\eta] v s . M_{w}$ as shown in Figure 1, has been ascribed to the increased branching of dextran with increasing molecular weight. It may be considered that the branching effect can explain the variations of $\bar{v}^{\circ}$ and $\Delta C_{p_{2}}{ }^{\circ}$ with molecular weight. First, we consider $\bar{v}_{2}^{\circ}$. The variation of specific volume $\bar{v}_{2}^{\circ}$, with molecular weight is a rather delicate problem. It has been shown ${ }^{12}$ that the partial specific volume of a polymer may be expressed by the relation,

$$
\bar{v}_{2}^{\circ}=\bar{v}_{m}+K / M_{n}
$$

where $K$ is a constant which is negative for polystyrene in benzene and positive for poly(methyl methacrylate) in benzene. ${ }^{12}$ This relation holds for polystyrenes with different end-groups, but only for those having $M_{n}$ below $4 \times 10^{4} .{ }^{13}$ For comb-type polystyrenes with the same degree of branching and the branches of identical length, the values of $\bar{v}_{2}{ }^{\circ}$ were independent of molecular weight. ${ }^{14}$ If these facts are considered, it is not certain whether the approximately constant values of $\bar{v}_{2}^{\circ}$ of dextran illustrated in Table III are related to the branching 
of the polymer chain. Next, we consider $\Delta \overline{C_{p_{2}}}$. The increase of branching will decrease the portion of the polymer chain in direct contact with the solvent, thus giving rise to the decrease in the parameter $\Delta \overline{C_{p_{2}}}$. However, the interpretation in terms of branching does not go well with the results for $\omega$. The reasons are as follows. As mentioned above, it is reasonable to assume that the amount of hydration determined in the present work corresponds to the bound water attracted by the hydrophilic group of $\mathrm{OH}$. If one branching is produced in the polymer chain, one $\mathrm{OH}$ group is decreased, or a result of the formation of $-\mathrm{O}-\mathrm{C}-$ linkage, but at the same time one end group $\mathrm{OH}$ appears; The total number of $\mathrm{OH}$ groups in the polymer chain remains unchanged. Therefore, the effect of branching is not the only factor governing the molecular weight dependence of the physical parameters of aqueous solutions of dextran. In addition to branching, effects of the entanglement of polymer chains should also be taken into account: With increasing molecular weight, the entanglement of polymer chains increases. This effect produces i) a decrease in the number of free $\mathrm{OH}$ groups available for hydration and ii) weakening of the interaction between solute-solvent with the result that the degree of the structural change upon dissolution of unit structure of polymer decreases.

It seems to be more appropriate to attribute the molecular weight dependences observed in this study to the combination of branching and en- tanglement of the polymer chains.

\section{REFERENCES}

1. H. Nomura, S. Yamaguchi, and Y. Miyahara, $J$. Appl. Polym. Sci., 8, 2731 (1964).

2. K. Gekko and H. Noguchi, Biopolymers, 10, 1513 (1964).

3. F. R. Senti, N. N. Hellman, N. H. Ludwig, G. E. Babcock, R. Tobin, C. A. Glass, and B. L. Lamberts, J. Polym. Sci., 17, 527 (1955).

4. K. A. Granath, J. Colloid Sci., 13, 308 (1958).

5. S. Kato, T. Suzumi, H. Nomura, and Y. Miyahara, Macromolecules, 13, 889 (1980).

6. Y. Miyahara, H. Nomura, F. Kawaizumi, and N. Nishio, Rep. Asahi Glass Found. Ind. Technol., 34, 273 (1979).

7. F. Kawaizumi, N. Nishio, H. Nomura, and Y. Miyahara, J. Chem. Thermodyn., 13, 89 (1981).

8. H. Nomura and Y. Miyahara, J. Appl. Polym. Sci., 8, 1643 (1964).

9. H. Shiio, J. Am. Chem. Soc., 80, 70 (1958).

10. C. J. Dobratz, Ind. Eng. Chem., 33, 757 (1941). The estimated values of $C_{p_{2}}$ of mono- and di-saccharides obtained by the present method are in excellent agreement with those found experimentally.

11. Calculated from the data of the apparent molar compressibility of the aqueous solutions of glucose give by H. Høiland and H. Holvik, J. Solution Chem., 7, 587 (1978).

12. G. V. Schulz and M. Hoffmann, Makromol. Chem., 23, 220 (1957).

13. J. François and F. Candau, Eur. Polym. J., 9, 1355 (1973).

14. F. Candau and J. François, C. R. Acad. Sci., Ser. C, 276, 571 (1973). 